\title{
Root Separation for Reducible Monic Quartics
}

\author{
Andrej Dujella (*) - Tomislav Pejković (**)
}

ABSTRACT - We study root separation for reducible monic integer polynomials of degree four. If $\mathrm{H}(P)$ is the height and $\operatorname{sep}(P)$ the minimal distance between two distinct roots of a separable integer polynomial $P(x)$, and $\operatorname{sep}(P)=\mathrm{H}(P)^{-e(P)}$, we show that $\lim \sup e(P)=2$, where limsup is taken over all reducible monic integer polynomials $P(x)$ of degree 4 .

\section{Introduction.}

The height $\mathrm{H}(P)$ of an integer polynomial $P(x)$ is the maximum of the absolute values of its coefficients. For an integer polynomial $P(x)$ of degree $d \geq 2$ and with distinct roots $\alpha_{1}, \ldots, \alpha_{d}$, we set

$$
\operatorname{sep}(P):=\min _{1 \leq i<j \leq d}\left|\alpha_{i}-\alpha_{j}\right|
$$

and define $e(P)$ by

$$
\operatorname{sep}(P):=\mathrm{H}(P)^{-e(P)} .
$$

For an infinite set $S$ of integer polynomials containing polynomials of arbitrary large height, we define

$$
e(S)=\limsup _{P(X) \in S, \mathrm{H}(P) \rightarrow+\infty} e(P) .
$$

(*) Indirizzo dell'A.: Department of Mathematics, University of Zagreb, Bijenička cesta 30, 10000 Zagreb, Croatia.

E-mail: duje@math.hr

(**) Indirizzo dell'A.: Tomislav Pejković, Department of Mathematics, University of Zagreb, Bijenička cesta 30, 10000 Zagreb, Croatia.

E-mail: pejkovic@math.hr

The authors were supported by the Ministry of Science, Education and Sports, Republic of Croatia, grant 037-0372781-2821 and by the French-Croatian bilateral COGITO project Diophantine approximations. 
In this note we will be concerned with reducible monic polynomials of degree four with integer coefficients. Therefore, we introduce notation $\mathcal{R} \mathcal{M}_{d}$ for the set of all reducible monic polynomials of degree $d$ with integer coefficients.

First, we briefly summarize what is known about bounds on $e(S)$ if $S$ is some class of integer polynomials of small degree. A classical result of Mahler [5] asserts that if $S$ contains only polynomials of degree $d$, then $e(S) \leq d-1$.

The case of quadratic polynomials is almost trivial and won't be discussed further:

\begin{tabular}{|c|c|c|}
\hline$d=2$ & general & monic \\
\hline irreducible & $e=1$ & $e=0$ \\
\hline reducible & $e=1$ & $e=0$ \\
\hline
\end{tabular}

For cubic polynomials, the case of general (i.e. nonmonic) polynomials was first solved by Evertse [4] and later Schönhage [6] gave an easier constructive proof. In the monic case Bugeaud and Mignotte [3] proved the lower bound $e\left(\mathcal{M}_{3}\right) \geq \frac{3}{2}$, where $\mathcal{M}_{3}$ is the set of monic cubic polynomials with integer coefficients. They also showed that $e\left(\mathcal{M}_{3}\right)=\frac{3}{2}$ is equivalent to Hall conjecture. Proving that $e\left(\mathcal{R} \mathcal{M}_{3}\right)=1$ is not hard when we notice that a polynomial from this set is a product of a linear and a quadratic polynomial, both monic and with integer coefficients because of Gauss's Lemma. In the next table we summarize known results for $d=3$ :

\begin{tabular}{|c|c|c|}
\hline$d=3$ & general & monic \\
\hline irreducible & $e=2$ & $e \geq \frac{3}{2}$ \\
\hline reducible & $e=2$ & $e=1$ \\
\hline
\end{tabular}

Until now no exact values when $d=4$ were known, just the lower bounds given in the following table:

\begin{tabular}{|c|c|c|}
\hline$d=4$ & general & monic \\
\hline irreducible & $e \geq \frac{13}{6}$ & $e \geq \frac{3}{2}$ \\
\hline reducible & $e \geq \frac{7}{3}$ & $e \geq 2$ \\
\hline
\end{tabular}


The bound for nonmonic irreducible case arises from a general construction by Bugeaud and Dujella [2] which in this special case gives $e\left(\left(\bar{P}_{4, n}(x)\right)_{n \in \mathbb{N}}\right)=$ $\frac{13}{6}$, where

$$
\bar{P}_{4, n}(x)=\left(20 n^{4}-2\right) x^{4}+\left(16 n^{5}+4 n\right) x^{3}+\left(16 n^{6}+4 n^{2}\right) x^{2}+8 n^{3} x+1 .
$$

For nonmonic reducible polynomials, a recent unpublished result by $\mathrm{Bu}-$ geaud and Dujella, shows that the sequence

$\widetilde{P}_{4, n}(x)=\left((2 n+1) x^{3}+(2 n-1) x^{2}+(n-1) x-1\right)\left(\left(n^{2}+3 n+1\right) x-(n+2)\right)$

gives $e \geq e\left(\left(\widetilde{P}_{4, n}(x)\right)_{n \in \mathbb{N}}\right)=\frac{7}{3}$. The bound for monic irreducible polynomials $e \geq \frac{3}{2}$ is deduced by looking at the sequence

$$
\widehat{P}_{4, n}(x)=\left(x^{2}-n x+1\right)^{2}-2(n x-1)^{2}, \quad n \in \mathbb{N}
$$

(see Bugeaud and Mignotte [3]). Finally, for reducible monic polynomials, it follows from a general case discussed in [3] that $e\left(\mathcal{R} \mathcal{M}_{4}\right) \geq 2$. While the proof from [3] is nonconstructive, in Section 2 we establish the same inequality by exhibiting a set $S \subseteq \mathcal{R} \mathcal{M}_{4}$ such that $e(S)=2$. In Section 3 we prove that $e\left(\mathcal{R} \mathcal{M}_{4}\right) \leq 2$. By putting together the results from Sections 2 and 3 , we obtain the main result of this paper, which gives the first exact value in the above table for $d=4$.

\section{THEOREM 1. It holds that $e\left(\mathcal{R} \mathcal{M}_{4}\right)=2$.}

Furthermore, in Section 4, we show that if the coefficients of polynomials in the sequence $S=\left(P_{n}(x)\right)_{n \in \mathrm{N}} \subseteq \mathcal{R} \mathcal{M}_{4}$ grow polynomially in $n$, we must have a strict inequality $e(S)<2$. But we also show that we can choose such a sequence so that $e(S)$ is arbitrarily close to 2 . More precisely, we prove the following theorem.

THEOREM 2. If $S=\left(P_{n}(x)\right)_{n \in \mathbb{N}} \subseteq \mathcal{R} \mathcal{M}_{4}$ is a sequence of polynomials whose coefficients are polynomials in $n$, then $e(S)<2$. For any $\varepsilon>0$, there is a a sequence of polynomials $S=\left(P_{n}(x)\right)_{n \in \mathbb{N}} \subseteq \mathcal{R} \mathcal{M}_{4}$ whose coefficients are polynomials in $n$ such that $e(S)>2-\varepsilon$.

A survey of results on separation of roots for integer polynomials of general degree can be found in the paper by Bugeaud and Mignotte [3] (see also [2]). 


\section{The constructive proof of $e\left(\mathcal{R} \mathcal{M}_{4}\right) \geq 2$.}

We want to find a sequence of polynomials $S=\left(P_{n}(x)\right)_{n \in \mathbb{N}} \subseteq \mathcal{R} \mathcal{M}_{4}$ such that $e(S)=2$. We look at integer polynomials of the type

$$
P(x)=\left(x^{2}+r x+s\right)\left(x^{2}+a x+b\right),
$$

where $r$ and $s$ are fixed while $a$ and $b$ depend on them and on $n$ such that one root of the polynomial in the first bracket is very close to a root of the polynomial in the second bracket.

Choose $r$ and $s$ such that the roots $\lambda_{1}, \lambda_{2}$ of the polynomial $R(x)=$ $x^{2}+r x+s \in Z[x]$ satisfy $\lambda=\lambda_{1}>1>\lambda_{2}>0$. Also, let $\left(a_{n}\right)_{n \in \mathbb{N}}$ be an increasing sequence of positive integers that satisfies the recurrence $a_{n+2}+r a_{n+1}+s a_{n}=0$ whose characteristic polynomial is $R(x)$. Hence,

$$
a_{n}=c_{1} \lambda_{1}^{n}+c_{2} \lambda_{2}^{n}=c_{1} \lambda^{n}+c_{2} \frac{s}{\lambda^{n}},
$$

for some constants $c_{1}, c_{2}$.

Assume that $\lambda+\varepsilon$ is a root of the polynomial $x^{2}+a x+b \in \mathbb{Z}[x]$. Then we have

$$
\begin{aligned}
(\lambda+\varepsilon)^{2}+a(\lambda+\varepsilon)+b & =0 \\
\varepsilon^{2}+(2 \lambda+a) \varepsilon+(a-r) \lambda+(b-s) & =0 .
\end{aligned}
$$

Therefore $2 \varepsilon=-(2 \lambda+a) \pm \sqrt{(2 \lambda+a)^{2}-4((a-r) \lambda+(b-s))}$. If we have

$$
2 \lambda+a>0 \quad \text { and } \quad|4((a-r) \lambda+(b-s))|<(2 \lambda+a)^{2},
$$

then we get a smaller $|\varepsilon|$ for the + sign, so

$$
\begin{array}{r}
|2 \varepsilon|=\left|\frac{4((a-r) \lambda+(b-s))}{-(2 \lambda+a)-\sqrt{(2 \lambda+a)^{2}-4((a-r) \lambda+(b-s))}}\right| \\
\asymp\left|\frac{(a-r) \lambda+(b-s)}{2 \lambda+a}\right|
\end{array}
$$

(here $M \asymp N$ stands for $M \ll N$ and $N \ll M$, where the implicit constants depend only on $r$ and $s$ ). At this point we see that by choosing

$$
a-r=a_{n}, \quad r \leq-1, \quad b-s=-a_{n+1}, \quad s=1,
$$

conditions on $\lambda_{1}, \lambda_{2},\left(a_{n}\right)_{n \in \mathbb{N}}$ and inequalities (1) are fulfilled, while from (2) 
we have

$$
\begin{array}{r}
\operatorname{sep}\left(P_{n}\right)=|\varepsilon| \asymp\left|\frac{a_{n} \lambda-a_{n+1}}{2 \lambda+a_{n}+r}\right|=\left|\frac{c_{1} \lambda^{n+1}+\frac{c_{2}}{\lambda^{n-1}}-c_{1} \lambda^{n+1}-\frac{c_{2}}{\lambda^{n+1}}}{2 \lambda+c_{1} \lambda^{n}+\frac{c_{2}}{\lambda^{n}}+r}\right| \\
\asymp \frac{1}{\lambda^{2 n}} \asymp \max \{1,|a|,|b|\}^{-2} \asymp \mathrm{H}\left(P_{n}\right)^{-2}
\end{array}
$$

and thus

$$
e\left(\left(P_{n}\right)_{n \in \mathbb{N}}\right)=2
$$

where

$$
P_{n}(x)=\left(x^{2}+r x+1\right)\left(x^{2}+\left(r+a_{n}\right) x+\left(1-a_{n+1}\right)\right) .
$$

This shows that $e\left(\mathcal{R} \mathcal{M}_{4}\right) \geq 2$.

Note that we could have taken $s=-1$ before and if we were trying to approach the smaller root i.e. $\lambda_{2}$, we would get a similar family of polynomials

$$
P_{n}(x)=\left(x^{2}+r x-1\right)\left(x^{2}+\left(r-a_{n+1}\right) x-\left(a_{n}+1\right)\right),
$$

and after substitution $x \mapsto-x$, we would get

$$
P_{n}(x)=\left(x^{2}-r x-1\right)\left(x^{2}+\left(-r+a_{n+1}\right) x-\left(a_{n}+1\right)\right) .
$$

In case of $a_{1}=1, a_{2}=1, r=-1$, the above polynomial is

$$
P_{n}(x)=\left(x^{2}+x-1\right)\left(x^{2}+\left(1+F_{n+1}\right) x-\left(F_{n}+1\right)\right)
$$

where $\left(F_{n}\right)_{n \in \mathbb{N}}$ is the Fibonacci sequence. This last sequence of polynomials, which was first obtained by numerical experiments, was the motivating factor for this study.

\section{The proof of $e\left(\mathcal{R} \mathcal{M}_{4}\right) \leq 2$.}

Let us prove that $e\left(\mathcal{R} \mathcal{M}_{4}\right) \leq 2$. In other words, the best separation of roots we can get in the case of a reducible separable monic quartic polynomial $P(x) \in Z[x]$ is $\asymp(\mathrm{H}(P))^{-2}$. (All the constants implied in $\asymp, \ll, \gg$ in this section are absolute.)

We have to look at two cases: when the polynomial has a cubic irreducible factor and when the polynomial has a quadratic irreducible factor. Because of Gauss's Lemma all the divisors in $\mathrm{Q}[x]$ of $P(x)$ will actually be from $Z[x]$. Therefore, the case when $P(x)$ is a product of linear factors is trivial. 
If we have $P(x)=(x-k)\left(x^{3}+a x^{2}+b x+c\right)$, where $a, b, c, k \in Z$, then by the result of Mahler we know that the roots of $Q(x)=x^{3}+a x^{2}+b x+c$ can be no closer than $\asymp(\max \{1,|a|,|b|,|c|\})^{-2}$. Because of Gelfond's Lemma (see e.g. [1, p. 221]), we have

(3) $\frac{1}{16} \max \{1,|k|\} \max \{1,|a|,|b|,|c|\}$

$$
\leq \mathrm{H}(P) \leq 16 \max \{1,|k|\} \max \{1,|a|,|b|,|c|\},
$$

so $\operatorname{sep}(Q) \gg \mathrm{H}(P)^{-2}$. There only remains to check whether we can have a root of $Q(x)$ close to $k$. Let us take $Q(k+\varepsilon)=(k+\varepsilon)^{3}+a(k+\varepsilon)^{2}+$ $b(k+\varepsilon)+c=0$ where without loss of generality we can suppose $|\varepsilon|<1$. It is obvious that $|k+\varepsilon|<|a|+|b|+|c|+1$ must hold, otherwise we get a contradiction. Thus, from (3) we get $|k| \ll \mathrm{H}(P)^{1 / 2}$. Since $P(x)$ does not have multiple roots and $Q(x) \in Z[x]$ we have

$$
1 \leq|Q(k)|=|Q(k+\varepsilon)-Q(k)|=\left|Q^{\prime}(t)\right| \cdot|\varepsilon|,
$$

where $t \in(k, k+\varepsilon) \subset(k-1, k+1)$. But, using (3) and $|k| \ll \mathrm{H}(P)^{1 / 2}$, we get

$$
\left|Q^{\prime}(t)\right|=\left|3 t^{2}+2 a t+b\right| \leq 3(|k|+1)^{2}+2|a|(|k|+1)+|b| \ll \mathrm{H}(P) .
$$

Finally, we arrive at $|\varepsilon| \geq 1 /\left|Q^{\prime}(t)\right| \gg \mathrm{H}(P)^{-1}$.

If $P(x)=Q_{1}(x) Q_{2}(x)$, where $Q_{1}(x), Q_{2}(x) \in Z[x]$ are two quadratic polynomials, then we have from Gelfond's Lemma

$$
\frac{1}{16} \mathrm{H}\left(Q_{1}\right) \mathrm{H}\left(Q_{2}\right) \leq \mathrm{H}(P) \leq 16 \mathrm{H}\left(Q_{1}\right) \mathrm{H}\left(Q_{2}\right) .
$$

Since for quadratic polynomials we have $\operatorname{sep}\left(Q_{i}\right) \gg H\left(Q_{i}\right)^{-1}$, we only have to check the proximity of the roots $\alpha$ and $\beta$ of $Q_{1}(x)$ and $Q_{2}(x)$, respectively. Theorem A.1 from [1, p. 223] states that in our separable case

$$
|\alpha-\beta| \geq 2^{-1} 3^{-5 / 2} \cdot \mathrm{H}\left(Q_{1}\right)^{-2} \mathrm{H}\left(Q_{2}\right)^{-2} \cdot \max \{1,|\alpha|\} \max \{1,|\beta|\} \gg \mathrm{H}(P)^{-2} .
$$

Hence, we proved that $e\left(\mathcal{R} \mathcal{M}_{4}\right) \leq 2$, which concludes the proof of Theorem 1.

\section{Polynomial growth of coefficients.}

In Section 2 we exhibited a family of reducible monic polynomials $P_{n}(x)$ whose coefficients grow exponentially in $n$ such that $\operatorname{sep}\left(P_{n}\right) \asymp \mathrm{H}\left(P_{n}\right)^{-2}$.

We will show that this is not possible if the coefficients grow polynomially. More precisely, let $P_{n}(x)=P(n, x) \in \mathbb{Z}[n, x]$ be a polynomial 
which is monic of degree 4 in $x$ and such that for every positive integer $n^{\prime}$, polynomial $P_{n^{\prime}}(x) \in Z[x]$ is reducible. This is the exact meaning of conditions in the first statement of Theorem 2. Hilbert's Irreducibility Theorem (see e.g. Zannier [7]) implies that

$$
P_{n}(x)=Q_{n, 1}(x) Q_{n, 2}(x),
$$

where $Q_{n, 1}(x)$ and $Q_{n, 2}(x)$ are monic polynomials in $x$ whose coefficients are integer polynomials in $n$. Note that because of the previous section, the case of a reducible monic polynomial with a linear factor is not very interesting. Therefore, we will assume that $Q_{n, 1}(x)$ and $Q_{n, 2}(x)$ are irreducible quadratic polynomials in $x$ without common roots, so

$$
Q_{n, 1}(x)=x^{2}+r(n) x+s(n), \quad Q_{n, 2}(x)=x^{2}+a(n) x+b(n),
$$

where $r(n), s(n), a(n), b(n) \in \mathbb{Z}[n]$. For the sake of simplicity, we will most often omit $n$. As already mentioned, we can assume that the closest roots of $P$ are a root of $Q_{1}$ and a root of $Q_{2}$. So, without loss of generality, let us take

$$
2 \operatorname{sep}(P)=2 \varepsilon=-r+\sqrt{r^{2}-4 s}+a+\sqrt{a^{2}-4 b} .
$$

After some manipulation we get that $\varepsilon$ satisfies the following equality

$$
\begin{aligned}
\varepsilon^{4}- & -2(a-r) \varepsilon^{3}+\left(r^{2}+a^{2}-3 r a+2 s+2 b\right) \varepsilon^{2} \\
& -(a-r)(-r a+2 s+2 b) \varepsilon+\left(s^{2}+b^{2}-r s a-r a b-2 b s+s a^{2}+b r^{2}\right)=0 .
\end{aligned}
$$

Notice that the last term is just the resultant $\operatorname{Res}_{x}\left(Q_{1}, Q_{2}\right)$ of polynomials $Q_{1}$ and $Q_{2}$ :

$$
\operatorname{Res}\left(Q_{1}, Q_{2}\right)=\operatorname{Res}\left(Q_{1}, Q_{2}-Q_{1}\right)=(b-s)^{2}+(a-r)(a s-b r) .
$$

Let us suppose that $\varepsilon \ll \mathrm{H}^{-2}$, where by Gelfond's Lemma $\mathrm{H}=\mathrm{H}(P) \asymp \mathrm{H}\left(Q_{1}\right) \mathrm{H}\left(Q_{2}\right)$. It can be mentioned here that all the constants in $\mathcal{O}, \ll, \gg, \asymp$ in the first part of this section depend at most on the coefficients of $r, s, a, b$. Since $P(x)$ is a separable integer polynomial, it follows that $\operatorname{Res}\left(Q_{1}, Q_{2}\right)$ is an integer polynomial in $n$ and $\left|\operatorname{Res}\left(Q_{1}, Q_{2}\right)\right| \geq 1$. Now we get from (5) and (4) that

$$
\mathrm{H}^{-2} \gg \varepsilon \gg \frac{\left|\operatorname{Res}\left(Q_{1}, Q_{2}\right)\right|}{|\underbrace{\varepsilon^{3}}_{\mathcal{O}\left(\mathrm{H}^{-6}\right)}-\underbrace{2(a-r) \varepsilon^{2}}_{\mathcal{O}\left(\mathrm{H}^{-3}\right)}+\underbrace{\underbrace{r^{2}+a^{2}}_{\mathcal{O}\left(\mathrm{H}^{2}\right)}-\underbrace{3 r a+2 s+2 b}_{\mathcal{O})}) \varepsilon}_{\mathcal{O}(1)}-(a-r)(-r a+2 s+2 b)|}
$$


and

$$
\mathrm{H}^{-2} \gg \varepsilon \gg \frac{\left|\operatorname{Res}\left(Q_{1}, Q_{2}\right)\right|}{|\mathcal{O}(1)-\underbrace{2 a s+2 r b}_{\mathcal{O}(\mathrm{H})}+r a^{2}-r^{2} a+2 r s-2 a b|} .
$$

Because of Gelfond's Lemma, $|r|,|s|,|a|,|b| \ll \mathrm{H}$ and $|a r| \ll \mathrm{H}$ which implies that $|a| \ll \mathrm{H}^{1 / 2}$ or $|r| \ll \mathrm{H}^{1 / 2}$. Without loss of generality we can suppose that $|a| \ll \mathrm{H}^{1 / 2}$. Thus we get $\left|r a^{2}\right|=|r a| \cdot|a| \ll \mathrm{H}^{3 / 2}$ and $|a b|=|a| \cdot|b| \ll \mathrm{H}^{3 / 2}$. We also have $\left|-r^{2} a+2 r s\right|=|r| \cdot|r a-2 s|=|r| \mathcal{O}(\mathrm{H})$ so the inequality (6) becomes

$$
\mathrm{H}^{-2} \gg \varepsilon \gg \frac{1}{\max \left\{\mathcal{O}\left(\mathrm{H}^{3 / 2}\right),|r| \mathcal{O}(\mathrm{H})\right\}}
$$

It implies that $|r| \gg \mathrm{H}$, so from $|r| \ll \mathrm{H}$, we get $|r| \asymp \mathrm{H}$. Also, $\left|\operatorname{Res}\left(Q_{1}, Q_{2}\right)\right|=\mathcal{O}(1)$. Since $r, s, a, b$ are polynomials in $n$ and $|r a| \ll \mathrm{H}$, $|r b| \ll \mathrm{H}$, we conclude that $a$ and $b$ are constants.

If we now have $\operatorname{deg}_{n} s<\operatorname{deg}_{n} r$ then

$$
\operatorname{deg}_{n} \operatorname{Res}\left(Q_{1}, Q_{2}\right)=\operatorname{deg}_{n}\left((b-s)^{2}+(a-r)(a s-b r)\right) \geq \operatorname{deg}_{n} r+\operatorname{deg}_{n} s
$$

so $\left|\operatorname{Res}\left(Q_{1}, Q_{2}\right)\right| \gg \mathrm{H}$, which leads to a contradiction. Therefore, $\operatorname{deg}_{n} s=$ $\operatorname{deg}_{n} r$ and hence $|s| \asymp|r| \asymp \mathrm{H} \rightarrow \infty$.

The leading coefficient of $\operatorname{Res}\left(Q_{1}, Q_{2}\right)$ as a polynomial in $n$, i.e. the coefficient that belongs to the monomial of degree $2 \operatorname{deg}_{n} r=2 \operatorname{deg}_{n} s$, is the leading coefficient of $s^{2}-a r s+b r^{2}$, i.e. $k_{s}^{2}-a k_{r} k_{s}+b k_{r}^{2}$, where $k_{s}$, $k_{r}$ are leading coefficients of $s$ and $r$, respectively. If it were 0 , then $-k_{s} / k_{r} \in \mathrm{Q}$ would be a root of $x^{2}+a x+b$ which is impossible, since by our assumption this polynomial is irreducible. Thus $\operatorname{deg}_{n} \operatorname{Res}\left(Q_{1}, Q_{2}\right)=$ $2 \operatorname{deg}_{n} r \geq 2$ and this is in contradiction with the condition $\left|\operatorname{Res}\left(Q_{1}, Q_{2}\right)\right|=$ $\mathcal{O}(1)$.

We conclude that $\operatorname{sep}\left(P_{n}\right) \ll \mathrm{H}\left(P_{n}\right)^{-2}$ cannot hold in this case, and this proves the first statement of Theorem 2 .

Although the previous result of this section shows that we cannot have a family of reducible monic quartic integer polynomials with polynomial growth of coefficients that has the best possible exponent for root separation in this case, i.e. -2 , we can still construct families with the exponent as close to -2 as we like. The construction that follows is similar to the one in Section 2.

We look at the family of polynomials $P_{k, n}(x)$ indexed with $n \in \mathbb{N}$ in variable $x$. As before, we will usually omit $n$ and write simply $P_{k}(x)$. We 
define

$$
\begin{aligned}
P_{k}(x) & =\underbrace{\left(x^{2}+n x+1\right)}_{Q_{k}(x)} \underbrace{\left(x^{2}+n x+1+A_{k+1} x+A_{k}\right)}_{R_{k}(x)} \\
& =(x^{2}+\underbrace{n}_{r} x+\underbrace{1}_{s})(x^{2}+\underbrace{\left(A_{k+1}+n\right)}_{a} x+\underbrace{\left(A_{k}+1\right)}_{b}),
\end{aligned}
$$

where $\left(A_{k}(n)\right)_{k \in \mathbb{N}_{0}}$ is defined recursively by

$$
A_{0}(n)=1, A_{1}(n)=n, \quad A_{k+1}(n)=n A_{k}(n)-A_{k-1}(n) \text { for } n \geq 2 .
$$

It is easy to see that $\operatorname{deg}_{n} A_{k}=k$, so we get (implied constants are absolute from now on)

$$
\mathrm{H}\left(P_{k}\right) \asymp n^{k+2} \text {. }
$$

Let us look at the resultant:

$$
\begin{aligned}
\operatorname{Res}_{x}\left(Q_{k}, R_{k}\right) & =(b-s)^{2}-r(b-s)(a-r)+s(a-r)^{2} \\
& =A_{k}^{2}-n A_{k} A_{k+1}+A_{k+1}^{2} \\
& =A_{k}^{2}+A_{k+1}\left(A_{k+1}-n A_{k}\right) \\
& =A_{k}^{2}-A_{k+1} A_{k-1} \\
& =A_{k}^{2}-\left(n A_{k}-A_{k-1}\right) A_{k-1} \\
& =A_{k}\left(A_{k}-n A_{k-1}\right)+A_{k-1}^{2} \\
& =A_{k-1}^{2}-A_{k} A_{k-2} \\
& =\ldots=A_{1}^{2}-A_{2} A_{0}=n^{2}-\left(n^{2}-1\right) \cdot 1=1 .
\end{aligned}
$$

The roots of $Q_{k}(x)$ are

$$
\alpha_{1}=\frac{-n-\sqrt{n^{2}-4}}{2}, \quad \alpha_{2}=\frac{-n+\sqrt{n^{2}-4}}{2},
$$

and the roots of $R_{k}(x)$ are

$$
\begin{aligned}
& \beta_{1}=\frac{-\left(A_{k+1}+n\right)-\sqrt{\left(A_{k+1}+n\right)^{2}-4\left(A_{k}+1\right)}}{2}, \\
& \beta_{2}=\frac{-\left(A_{k+1}+n\right)+\sqrt{\left(A_{k+1}+n\right)^{2}-4\left(A_{k}+1\right)}}{2} .
\end{aligned}
$$

Therefore,

$$
\alpha_{1} \asymp-n, \quad \alpha_{2} \asymp-\frac{1}{n}, \quad \beta_{1} \asymp-n^{k+1}, \quad \beta_{2}=\frac{A_{k}+1}{\beta_{1}} \asymp \frac{-1}{n},
$$


so we have

$$
1=\operatorname{Res}\left(Q_{k}, R_{k}\right)=1^{2} 1^{2} \underbrace{\left|\alpha_{1}-\beta_{2}\right|}_{\asymp n} \underbrace{\left|\alpha_{1}-\beta_{1}\right|}_{\asymp n^{k+1}} \underbrace{\left|\alpha_{2}-\beta_{1}\right|}_{\asymp n^{k+1}} \operatorname{sep}\left(P_{k}\right),
$$

and it follows that

$$
\operatorname{sep}\left(P_{k}\right) \asymp n^{-2 k-3}=n^{-2(k+2)} n \asymp \mathrm{H}\left(P_{k}\right)^{-2+\frac{1}{k+2}} .
$$

Hence, we proved the last statement of Theorem 2.

Acknowledgements. The authors would like to thank Yann Bugeaud for introducing them to this topic and for useful comments on the previous version of this paper.

\section{REFERENCES}

[1] Y. Bugeaud, Approximation by algebraic numbers. Cambridge Tracts in Mathematics, Cambridge, 2004.

[2] Y. Bugeaud - A. Dujella, Root separation for irreducible integer polynomials, Bull. Lond. Math. Soc., to appear.

[3] Y. Bugeaud - M. Mignotte, Polynomial root separation, Intern. J. Number Theory, 6 (2010), pp. 587-602.

[4] J.-H. Evertse, Distances between the conjugates of an algebraic number, Publ. Math. Debrecen, 65 (2004), pp. 323-340.

[5] K. MAHLER, An inequality for the discriminant of a polynomial, Michigan Math. J., 11 (1964), pp. 257-262.

[6] A. Schönhage, Polynomial root separation examples, J. Symbolic Comput., 41 (2006), pp. 1080-1090.

[7] U. Zannier, On the Hilbert irreducibility theorem, Rend. Semin. Mat. Univ. Politec. Torino, 67, no. 1 (2009), pp. 1-14.

Manoscritto pervenuto in redazione il 28 ottobre 2010. 